\title{
GONZALO GALIANO
}

MARK ADRIAAN PELETIER

\section{Spatial localization for a general reaction- diffusion system}

Annales de la faculté des sciences de Toulouse $6^{e}$ série, tome $7, \mathrm{n}^{\circ} 3$ (1998), p. 419-441

<http://www.numdam.org/item?id=AFST_1998_6_7_3_419_0>

(C) Université Paul Sabatier, 1998, tous droits réservés.

L'accès aux archives de la revue «Annales de la faculté des sciences de Toulouse » (http://picard.ups-tlse.fr/ annales/) implique l'accord avec les conditions générales d'utilisation (http://www.numdam.org/conditions). Toute utilisation commerciale ou impression systématique est constitutive d'une infraction pénale. Toute copie ou impression de ce fichier doit contenir la présente mention de copyright.

\section{NumDam}

Article numérisé dans le cadre du programme

Numérisation de documents anciens mathématiques

http://www.numdam.org/ 


\title{
Spatial Localization for a General Reaction-Diffusion System ${ }^{(*)}$
}

\author{
Gonzalo Galiano(1) and Mark AdriaAn Peletier(2)
}

\begin{abstract}
RÉSUMÉ. - Nous appliquons une méthode d'énergie locale pour démontrer la localisation en espace du support des solutions d'un système général de réaction-diffusion. Nous démontrons que la vitesse de propagation est finie et qu'il existe des "temps de réponse" (waiting times) sous des hypothèses faibles sur la structure du système. Ces hypothèses admettent des termes de réaction additifs et multiplicatifs, une dépendance en temps et en espace des coefficients ainsi qu'un terme de convection de divergence nulle.
\end{abstract}

Abstract. - We use a local energy method to study the spatial localization of the supports of the solutions of a reaction-diffusion system with nonlinear diffusion and a general reaction term. We establish finite speed of propagation and the existence of waiting times under a set of weak assumptions on the structural form of the system. These assumptions allow for additive and multiplicative reaction terms and space- and time-dependence of the coefficients, as well as a divergence-free convection term.

\section{Introduction}

In the study of the transport of a chemical species through groundwater flow the following system of equations arises:

$$
\left\{\begin{array}{l}
\phi(u)_{t}+v_{t}-\operatorname{div} \mathbf{A}(u, \nabla u)+B(u, \nabla u)=0 \\
v_{t}=f(u, v) .
\end{array}\right.
$$

(*) Reçu le 21 août 1996, accepté le 5 novembre 1996

(1) Depto. Matematicas, Fac. Ciencias, Universidad de Oviedo, E-33007 Oviedo (Spain)

$$
\text { E-mail : galiano@orion.ciencias.uniovi.es }
$$

(2) Centrum voor Wiskunde en Informatica, P.O. Box 94079, NL-1090 GB Amsterdam (Netherlands)

E-mail : mark.peletier@cwi.nl 
The unknown functions $u$ and $v$ represent concentrations of the species; the former, in solution, and the latter in adsorption on the surface of the soil particles. The functions $\phi, \mathbf{A}, B$, and $f$ are all supposed given. In Section 3.1 we give a brief derivation of these equations.

Our interest lies in the support of solutions $(u, v)$ of (1.1). It is well known that solutions of the equation

$$
\phi(u)_{t}-\Delta u=0
$$

can exhibit a compact (spatial) support, provided the nonlinearity $\phi$ is degenerate at $u=0$. A travelling wave analysis of (1.1) (cf. [7]) shows that solutions with compact support can also exist when $\phi$ and the diffusion operator are non-degenerate; in that case the function $f$ must have a degeneracy at $u=0$. Another fact that is known from the literature is that interfaces of solutions of (1.2) can remain stationary for short time, before starting to move (and never stopping afterwards). This is called the waiting time phenomenon. In this article we wish to investigate the compact support and waiting time phenomena of solutions of (1.1) in a general way.

A number of results concerning finite speed of propagation for systems of equations are known in the literature. Díaz and Stakgold [5] considered problem (1.1) with a reaction term of the form $f(u, v)=g(u) h(v)$, and Hilhorst, Van der Hout, and L. A. Peletier [8] extended this to more general reaction terms. Both works consider one-dimensional autonomous situations with simple initial and boundary data. In addition, they heavily rely on the existence of a comparison principle.

The method that we use, often called "the energy method for free boundary problems", was introduced by Antontsev [3], rendered in a mathematically rigorous form by Díaz and Véron [6], and later extended and applied by several other authors, amongst whom Bernis [4] and Shmarev [10]. We refer to [1] and [2] for a good overview of the existing literature on this point. The method has two principal features. On one hand, it is a local method: it operates in subsets of the domain without taking into account global information like boundary conditions or boundedness of the domain. On the other hand, it has a very general setting, allowing to consider, for instance, problems in any space dimension, $(x, t)$-dependence of the different terms of the equations, and anisotropy. In addition we do not require a comparison principle. It is worth noting that the method is essentially qualitative, in the sense that it does not provide, in general, quantitative estimates of the evolution of the support. 
Let us start by giving the definitions of the properties of system (1.1) that we will prove in this article:

DEFINITION 1.1. - (Finite speed of propagation, FSP) If $(u, v)$ is a solution such that $u(\cdot, 0)$ and $v(\cdot, 0)$ both vanish on a ball $B\left(x_{0}, \rho_{0}\right)$, then there exists an instant $t_{0}>0$ and a continuous function $\rho:\left[0, t_{0}\right] \rightarrow \mathbb{R}$, $\rho(0)=\rho_{0}$, such that $u(\cdot, t)$ and $v(\cdot, t)$ vanish almost everywhere in $B\left(x_{0}, \rho(t)\right)$ for all $t \leq t_{0}$.

DEFINITION 1.2. - (Waiting times, WT) If $(u, v)$ is a solution such that $u(\cdot, 0)$ and $v(\cdot, 0)$ both vanish on a ball $B\left(x_{0}, \rho_{0}\right)$ and satisfy a flatness condition in an annulus $B\left(x_{0}, \rho_{1}\right) \backslash \overline{B\left(x_{0}, \rho_{0}\right)}$, then there exists an instant $t^{*}>0$ such that $u(\cdot, t)$ and $v(\cdot, t)$ vanish almost everywhere in $B\left(x_{0}, \rho_{0}\right)$ for all $t \in\left[0, t^{*}\right]$.

Two remarks are due. First, we have not yet defined the notion of a solution; we postpone this to Section 2.2. Second, the flatness condition that the defintion of the WT property refers to is unspecified; in practice, the precise condition depends on the assumptions that we make about the components of (1.1). This will become clear in what follows.

\subsection{Statement of results}

Let us state our hypotheses. We consider the problem

$$
\begin{cases}\phi(u)_{t}+v_{t}-\operatorname{div} \mathbf{A}(x, t, u, \nabla u)+B(x, t, u, \nabla u)=0 & (x, t) \in Q \\ v_{t}=f(x, t, u, v) & (x, t) \in Q \\ (u, v)=\left(u_{0}, v_{0}\right) & \text { at } t=0\end{cases}
$$

on a domain $Q=\Omega \times(0, T]$, where $\Omega$ is a bounded domain in $\mathbb{R}^{N}$. Solutions to this problem are defined in Definition 2.1. The following hypotheses shall hold throughout this article, even when not stated explicitly:

(1) $u_{0}, v_{0} \in L^{\infty}(\Omega)$;

(2) $\phi, \mathbf{A}, B$, and $f$ are Carathéodory functions;

(3) $m_{0} u^{p+1} \leq \Phi(u) \leq m_{1} u^{p+1}$ for all $u \geq 0$, where the function $\Phi$ is given by

$$
\Phi(u)=\int_{0}^{u} s \phi^{\prime}(s) \mathrm{d} s .
$$

(4) $\mathbf{A}(\cdot, \cdot, \cdot, \xi) \cdot \xi \geq m_{2}|\xi|^{2}, \xi \in \mathbb{R}^{N}$; 
(5) $|\mathbf{A}(\cdot, \cdot, \cdot, \xi)| \leq m_{3}|\xi|, \xi \in \mathbb{R}^{N}$;

(6) $B$ satisfies either

$$
B=0
$$

or

$$
B=\mathbf{w} \cdot \nabla \phi(u),
$$

where $\mathbf{w} \in L^{\infty}\left(Q ; \mathbb{R}^{N}\right)$ and $\operatorname{div} \mathbf{w}=0$.

(7) $f$ is Lipschitz continuous in the second variable:

$$
\left|f\left(x, t, u, v_{1}\right)-f\left(x, t, u, v_{2}\right)\right| \leq L\left|v_{1}-v_{2}\right|
$$

for all $u, v_{1}, v_{2} \in \mathbb{R}$, and $(x, t) \in Q$.

Here $0<p \leq 1$ and the numbers $m_{i}$ are positive constants. In view of properties FSP and WT defined previously we fix once and for all $x_{0} \in \Omega$ and $\rho_{0}>0$ such that $B_{\rho_{0}}=B\left(x_{0}, \rho_{0}\right) \subset \Omega$ and $u_{0}=v_{0}=0$ on $B_{\rho_{0}}$. In addition, for property WT we assume that $\rho_{1}>\rho_{0}$ and $B_{\rho_{1}} \subset \Omega$. We shall use the notation " $B_{\rho}$ " for $B\left(x_{0}, \rho\right)$.

We shall use the following hypotheses in the formulation of the different theorems:

(H1) There exists a number $0<\bar{v} \leq \infty$ and a non-negative function $\psi:[0, \bar{v}) \rightarrow \mathbb{R}$ such that

$$
(u-\psi(v)) f(x, t, u, v) \geq 0
$$

for all $u \geq 0,0 \leq v<\bar{v}, x \in B_{\bar{\rho}}$ and for all $t>0$. Here $\bar{\rho}>\rho_{0}$ and, if appropriate, $\bar{\rho}>\rho_{1}$. If $\bar{v}<\infty$ then we set $\psi(v)=\infty$ for all $v \geq \bar{v}$.

(H2) $0 \leq f(\cdot, \cdot, u, 0) \leq k_{1} u^{p}$ for all $u \geq 0$;

(H3) $k_{2} u^{\gamma} \leq f(\cdot, \cdot, u, 0) \leq k_{3} u^{\gamma}$ for all $u \geq 0$.

Here the exponent $p$ is the same as above and the exponent $\gamma$ is free to be chosen in $(0,1)$. The $k_{i}$ are positive constants. Whenever possible we shall omit the variables $x, t$ in expressions of the type $\mathbf{A}(x, t, u, \nabla u)$.

Although at first sight hypothesis (H1) may seem far-fetched, it arises in a natural way in the derivation of the model underlying (1.3). In Appendix 3.1 , we therefore give an outline of the derivation of the model and its relation to this hypothesis. 
To make clear what we shall state from hypothesis (H2) and (H3) let us assume for a moment that $f(u, 0, \cdot, \cdot)$ is a power-like function depending only on $u, f(u, 0)=u^{q}$, and that $u, v \in L^{\infty}(Q)$. Then we can restrict our attention to the case $0 \leq u, v \leq 1$ for which we shall prove the FSP and WT properties in the following range of parameters $p, q$ :

$$
\left.\begin{array}{l}
p=1, \quad 0<q<1 \text { (by means of (H3)) } \\
p<1, \quad p \leq q<\infty(\text { by means of }(\mathrm{H} 2)) \\
p<1, \quad 0<q<1 \text { (by means of (H3)) }
\end{array}\right\} \Rightarrow p<1, \quad 0<q<\infty .
$$

Therefore, we shall arrive at the desired results by the natural hypothesis of either strong absorption (even without parabolic degeneracy of our PDE), or parabolic degeneracy without restriction in the absorption.

We shall now state our main results. The first one extends a known result for the "porous medium equation" (1.2): if $p<1$, then, under a weak condition on $f$, system (1.3) has property FSP. Besides, an advection term of the form (1.5) does not change this property.

THEOREM A. - Let hypothesis (H1) be satisfied. If $p<1$ and the condition on the advective term (1.5) holds, then problem (1.3) has property FSP.

For the theorem on waiting times we introduce an auxiliary function:

$$
\Psi(s)=\int_{0}^{s} \psi(\sigma) \mathrm{d} \sigma
$$

where $\psi$ is given by (H1). If $s \geq \bar{v}$, then $\Psi(s)$ is taken equal to infinity.

THEOREM B. - Let hypothesis (H1) be satisfied and suppose that $B=0$. If $p<1$ then problem (1.3) has property WT. The accompanying flatness condition reads:

$$
\left\{\begin{array}{l}
\text { there exists a constant } C>0 \text { such that } \\
\int_{B_{\rho}} \Phi\left(u_{0}\right)+\int_{B_{\rho}} \Psi\left(v_{0}\right) \leq C\left(\rho-\rho_{0}\right)_{+}^{1 /(1-\beta)} \text { for all } 0<\rho<\rho_{1} .
\end{array}\right.
$$

Here $\beta$ is given by (2.10). 


\section{G. Galiano and M. A. Peletier}

It was already known from a travelling wave analysis [7] that if the function $f$ satisfies a certain kind of degeneracy, then finite speed of propagation can occur even for regular (i.e., Lipschitz continuous) nonlinearities $\phi$. The following theorem makes this statement precise.

THEOREM C. - Let either of the following conditions be satisfied:

$$
\text { (H2) with } p<1 \text { or (H马) with } \gamma<1 \text {. }
$$

Set $\eta=p$ for (H2) and $\eta=\gamma$ for (H马). Then,

(1) if the advection condition (1.5) is satisfied, then problem (1.3) has the property FSP;

(2) if $B=0$, then problem (1.3) also has the property WT under the assumption of the flatness condition:

$$
\left\{\begin{array}{c}
\text { there exists a constant } C>0 \text { such that } \\
\int_{B\left(x_{0}, \rho\right)} u_{0}^{\eta+1}+\int_{B\left(x_{0}, \rho\right)} v_{0}^{(\eta+1) / \eta} \leq \\
\leq C\left(\rho-\rho_{0}\right)_{+}^{1 /(1-\beta)} \text { for all } 0<\rho<\rho_{1} .
\end{array}\right.
$$

Here $\beta$ is given by (2.10) for (H2) and by (2.25) for (H3).

\subsection{A comparison with the method of travelling waves}

In many cases results of the type of Theorem A (finite speed of propagation) are proved by comparing the solution with travelling waves. This allows the often detailed information that can be obtained on travelling waves to be transferred to general solutions.

For this method to apply it is however necessary that the problem is autonomous, i.e., invariant under translations. Although the study of such equations and systems can lead to valuable insight, many applications explicitly require results that remain valid when this spatial invariance condition is relaxed. A typical example is the model of transport of chemical substances through a porous medium that is derived in Section 3.1. Even if the medium itself is supposed homogeneous, together with its characteristics such as the functions $\phi$ and $f$, then the dispersion coefficient $D$ will generally depend on space and time, because it depends on the discharge field $\mathbf{w}$. 
Simply stated, $D$ is a tensor that "has the value" $\alpha_{L}|\mathbf{w}|$ in the direction of $\mathbf{w}$ and $\alpha_{T}|\mathbf{w}|$ in the other directions. Note that such a dispersion coefficient does not only bring space- and time-dependence into the problem, but also anisotropy.

The price to be paid for the gain in generality, however, is obvious; for instance, while for the travelling wave solutions (necessarily in homogeneous media) that were examined in [7] a very precise characterization could be given of the occurrence and non-occurrence of bounded supports, in the general case we just obtain the one-sided results of Theorems A, B and C.

For the travelling wave method it is also necessary that the problem satisfy a comparison principle. It is not difficult to see that if $B=0$ and $\Phi$ is an increasing function, system (1.1) satisfies a comparison principle if and only if $f$ is increasing in $u$ and decreasing in $v$. A comparison principle is a very important tool, not only in proving existence and uniqueness, but also in proving finite speed of propagation (property FSP) or its converse, by comparing the solution with travelling waves. Generically, the information about the travelling waves immediately carries over to the full problem.

Therefore it is important to note that the conditions that we set on $f$ allow for non-monotonicity. Indeed, they could be said to imply a form of "weak monotonicity": a function $f$ that is increasing in $u$ and decreasing in $v$ automatically satisfies condition ( $\mathrm{H} 1)$ as well as the requirement

$$
f(u, 0) \geq 0 \text { for all } u>0
$$

which is part of (H2) and (H3).

As a second point of difference, we should note that in general a travelling wave comparison method can not prove the existence of waiting times, since travelling waves mostly have non-zero speed.

\section{Main results}

\subsection{An outline of the method}

As was mentioned in the Introduction, an important aspect of this energy method is its applicability to very general equations and systems. The other side of the coin is that proofs tend to be very technical and 
obscure the underlying ideas. We therefore start with an introduction to the method, aimed at conveying philosophy rather than mathematically correct statements. After this introduction we shall prove Theorems A, B and $\mathrm{C}$ in full rigor.

We shall discuss the method applied to a simplified version of (1.3):

$$
\left\{\begin{array}{l}
u_{t}^{p}+v_{t}-\Delta u=0 \\
v_{t}=f(u, v)
\end{array}\right.
$$

The result we seek is that of Theorem A, i.e., if $p<1$ and $f$ satisfies (H1), then system (2.1) has property FSP.

The key idea is to derive an ordinary differential inequality from this system of partial differential equations and to conclude by means of the study of this inequality. Following the definition of property FSP we assume that the initial data $u_{0}$ and $v_{0}$ both vanish in the ball $B_{\rho_{0}}=B\left(x_{0}, \rho_{0}\right)$. We multiply the first equation of (2.1) by the solution $u$ and integrate by parts on a ball $B_{\rho}$ centered in $x_{0}$ with radius $\rho<\rho_{0}$. We obtain

$$
\frac{p}{p+1} \frac{\mathrm{d}}{\mathrm{d} t} \int_{B_{\rho}} u^{p+1}+\int_{B_{\rho}}|\nabla u|^{2}=\int_{\partial B_{\rho}} u \nabla u \cdot \nu-\int_{B_{\rho}} u f(u, v) .
$$

By integrating over $(0, t)$ for some $0<t<T$,

$$
\frac{p}{p+1} \int_{B_{\rho}} u(t)^{p+1}+\int_{0}^{t} \int_{B_{\rho}}|\nabla u|^{2}=\int_{0}^{t} \int_{\partial B_{\rho}} u \nabla u \cdot \nu-\int_{0}^{t} \int_{B_{\rho}} u f(u, v) .
$$

We now define the non-negative functions $b$ and $E$, which represent generalized energies (whence the term "energy method"):

$$
b(\rho, t)=\sup _{0 \leq \tau \leq t} \int_{B_{\rho}} u(\tau)^{p+1} \text { and } E(\rho, t)=\int_{0}^{t} \int_{B_{\rho}}|\nabla u|^{2} .
$$

Both are non-decreasing with respect to $\rho$; using

$$
\frac{\partial E}{\partial \rho}(\rho, t)=\int_{0}^{t} \int_{\partial B_{\rho}}|\nabla u|^{2} \quad \text { for } \rho<\rho_{0}
$$

it follows from the Hölder inequality that

$$
\begin{aligned}
\int_{0}^{t} \int_{\partial B_{\rho}} u \nabla u \cdot \nu \leq & \left(\int_{0}^{t} \int_{\partial B_{\rho}} u^{2}\right)^{1 / 2}\left(\frac{\partial E}{\partial \rho}\right)^{1 / 2} . \\
& -426-
\end{aligned}
$$


Using this in (2.3) we find that

$$
\frac{p}{p+1} b(\rho, t)+E(\rho, t) \leq\left(\int_{0}^{t} \int_{\partial B_{\rho}} u^{2}\right)^{1 / 2}\left(\frac{\partial E}{\partial \rho}\right)^{1 / 2}-\int_{0}^{t} \int_{B_{\rho}} u f(u, v) .
$$

The next step consists in using an interpolation-trace inequality (see Appendix 3.3) to derive the estimate

$$
\left(\int_{0}^{t} \int_{\partial B_{\rho}} u^{2}\right)^{1 / 2} \leq C t^{(1-\theta) / 2} K(T)(E+b)^{\kappa}
$$

where

$$
\begin{gathered}
\kappa=\frac{\theta}{2}+\frac{1-\theta}{p+1}, \\
K(T)=\max \left(1, T^{\theta / 2}\right) \max \left(1, b\left(T, \rho_{1}\right)^{\theta(1-p) /(2(p+1))}\right),
\end{gathered}
$$

and

$$
\theta=\frac{N(1-p)+p+1}{N(1-p)+2 p+2}
$$

It is important to remark here that $\kappa>1 / 2$ if and only if $p<1$. Therefore the arguments that follow can only be executed if $p<1$, since they require that $\kappa>1 / 2$. This is the point in the reasoning where the degeneracy of the nonlinearity is essential. Applying Young's inequality we obtain from (2.5) that

$$
\begin{aligned}
& \left(\int_{0}^{t} \int_{\partial B_{\rho}} u^{2}\right)^{1 / 2}\left(\frac{\partial E}{\partial \rho}\right)^{1 / 2} \leq \\
& \quad \leq C t^{(1-\theta) / 2} K(T)(E+b)^{\kappa}\left(\frac{\partial E}{\partial \rho}\right)^{1 / 2} \\
& \quad \leq \varepsilon(E+b)+C_{\varepsilon}\left(C t^{(1-\theta) / 2} K(T)\right)^{1 /(1-\kappa)}\left(\frac{\partial E}{\partial \rho}\right)^{1 / \beta}
\end{aligned}
$$

where $0<\varepsilon<p /(p+1)$ and

$$
\begin{aligned}
\beta=2(1-\kappa) & =\frac{3 p+1+N(1-p)}{2 p+2+N(1-p)} . \\
& -427-
\end{aligned}
$$


By combining (2.6) and (2.9) we obtain

$$
b+E \leq C_{1} t^{(1-\theta) / \beta}\left(\frac{\partial E}{\partial \rho}\right)^{1 / \beta}-C_{2} \int_{0}^{t} \int_{B_{\rho}} u f(u, v),
$$

where $C_{1}$ and $C_{2}$ collect all the different constants.

In the rigorous proofs we shall show how to handle the second term on the right-hand side in (2.11) depending on the assumptions on $f$. For the moment we assume that it is non-positive, allowing us to find an ordinary differential inequality for the function $E$ :

$$
E(\rho, t) \leq C_{1} t^{(1-\theta) / \beta}\left(\frac{\partial E}{\partial \rho}\right)^{1 / \beta}(\rho, t),
$$

which holds for all $0<\rho<\rho_{0}$ and $0<t<T$. For such ordinary differential inequalities it is not difficult to prove the following lemma, which is a special case of Lemma 3.1, part 2 .

LEMma. - Let $\nu>0$ and $\beta<1$, and let $\psi \in C\left([0, T] \times\left[0, \rho_{0}\right]\right)$ be a non-negative function such that

$$
\psi^{\beta}(t, \rho) \leq K t^{\nu} \frac{\partial \psi}{\partial \rho}(t, \rho)
$$

for almost every $\rho \in\left(0, \rho_{0}\right)$ and for all $t \in[0, T]$. Then there exists a time $t^{*} \leq T$ and a continuous function $r:\left[0, t^{*}\right] \rightarrow \mathbb{R}$ with $r(0)=\rho_{0}$ such that

$$
\psi(\rho, t)=0 \quad \text { for all } \rho \text { and } t \text { such that } \rho \leq r(t) .
$$

We conclude from this Lemma that in the region $\rho \leq r(t)$ the function $E(t, \rho)$, and therefore also the function $b(t, \rho)$, is equal to zero. Therefore system (2.1) possesses property FSP.

As we said above, this is not more than an intuitive outline of a general method which can be proved in full rigour. Many steps are only formal, and the treatment of the function $f$ has been completely neglected. In Sections $2.2,2.3$ and 2.4 , we shall give the details which are necessary to render the ideas presented above rigorous. 


\subsection{Proof of Theorem A}

Even for the simple system (2.1) the proof of Theorem A given above is not complete. The steps that were omitted were:

(1) the definition of a solution of the problem;

(2) the justification of equations (2.2) and (2.4) and estimate (2.5). This depends strongly on the choice of the function space to which a solution $(u, v)$ should belong;

(3) the treatment of the term $\int_{0}^{t} \int_{B_{\rho}} u f(u, v)$.

Besides, in order to complete the proof for system (1.3), we need to consider the more general functions $\phi, \mathbf{A}, B$, and $f$ instead of their simple counterparts in (2.1). We shall discuss these points one by one.

The definition of a solution. - This first omission is easily remedied.

Definition 2.1. - A pair of measurable functions $(u, v)$ defined in $Q=\Omega \times(0, T]$ is a weak solution of (1.3) with initial data $\left(u_{0}, v_{0}\right)$ if the following conditions are satisfied:

(1) $u \in L^{\infty}\left(0, T ; L^{p+1}(\Omega)\right) \cap L^{2}\left(0, T ; H^{1}(\Omega)\right)$ and $v \in L^{\infty}(Q)$;

(2) $u \geq 0$ and $v \geq 0$;

(3) $\mathbf{A}(\cdot, \cdot, u, \nabla u) \in L^{1}\left(Q ; \mathbb{R}^{N}\right)$ and $B(\cdot, \cdot, u, \nabla u) \in L^{1}(Q)$;

(4) $\varliminf_{t \rightarrow 0} \Phi(u(\cdot, t))=\Phi\left(u_{0}\right)$ and $\varliminf_{t \rightarrow 0} v(\cdot, t)=v_{0}$ in $L^{1}(\Omega)$;

(5) for any $\psi \in C^{\infty}\left([0, T] ; C_{c}^{\infty}(\Omega)\right)$ we have

$$
\begin{gathered}
\int_{\Omega} \Phi(u) \psi(T)-\int_{Q}\left\{\Phi(u) \psi_{t}-\mathbf{A}(u, \nabla u) \cdot \nabla \psi-B(u, \nabla u) \psi\right\}= \\
=\int_{\Omega} \Phi\left(u_{0}\right) \psi(0)-\int_{Q} f(u, v) \psi
\end{gathered}
$$

and

$$
\int_{\Omega} v \psi(T)-\int_{Q} v \psi_{t}=\int_{\Omega} v_{0} \psi(0)+\int_{Q} f(u, v) \psi,
$$

where we have omitted the variable pair $(x, t)$ for clarity.

We emphasize that we leave aside all questions of uniqueness, as well as existence under given boundary conditions. The arguments that follow only require the existence of a solution in the local sense of Definition 2.1. 
Non-zero convection. - In order to accommodate non-zero convection (condition (1.5)) we shall use a domain of integration that is not the cylinder $B_{\rho} \times(0, T)$ but a truncated cone

$$
K_{\rho, t}=\{(x, \tau) \in \Omega \times(0, t):|x|<g(\rho, \tau)\},
$$

where $g(\rho, \tau):=\rho-\alpha \tau$ and $\alpha>0$ shall be fixed later. For a general function $\psi$, we introduce the notation

$$
\int_{K_{\rho, t}} \psi \equiv \int_{0}^{t} \int_{B_{g}} \psi \equiv \int_{0}^{t} \int_{B_{g(\rho, \tau)}} \psi \mathrm{d} x \mathrm{~d} \tau
$$

and similarly for the boundary integrals.

It can easily be verified that the following identity holds for smooth functions $\zeta$ :

$$
\int_{B_{g(\rho, t)}} \frac{\partial \zeta}{\partial t}(x, t) \mathrm{d} x=\frac{\mathrm{d}}{\mathrm{d} t} \int_{B_{g(\rho, t)}} \zeta(x, t) \mathrm{d} x-g^{\prime}(t) \int_{\partial B_{g(\rho, t)}} \zeta(x, t) \mathrm{d} s .
$$

By a truncation-regularization scheme such as in [1] or [6], we can combine this formula with equation (2.13) (for $\psi=u$ ) to obtain for all $0<\rho<\rho_{0}$ and for all $0<t<T$,

$$
\begin{gathered}
\left.\int_{B_{g(\rho, t)}} \Phi(u(t))+\int_{0}^{t} \int_{\partial B_{g}}(\mathbf{w} \cdot \nu+\alpha)\right) \Phi(u)+\int_{K_{\rho, t}} \mathbf{A}(u, \nabla u) \cdot \nabla u= \\
=\int_{0}^{t} \int_{\partial B_{g}} u \mathbf{A}(u, \nabla u) \cdot \nu-\int_{K_{\rho, t}} u f(u, v) .
\end{gathered}
$$

When $\rho$ is allowed to take values in the interval $\left(\rho_{0}, \rho_{1}\right)$, as is necessary for property WT, the right-hand side of (2.16) contains the extra term $\int_{B_{\rho}} \Phi\left(u_{0}\right)$.

Equation (2.16) is the equivalent of (2.2) for general functions $\phi, \mathbf{A}$, and $B$. Observe that by choosing $\alpha=\|\mathbf{w}\|_{L^{\infty}(Q)}$, the second term on the left-hand side becomes non-negative.

Remark. - The use of a cone instead of a cylinder has a very simple physical interpretation. When $\alpha=\|\mathbf{w}\|_{L^{\infty}(Q)}$, the spatial boundary of the cone (i.e., $\left.\partial B_{g(\rho, t)}\right)$ moves inward with time with a velocity that is as least as large as the maximum velocity of the flow field. Therefore 
the convection term will not introduce any material from outside into the integration domain; the occurrence or non-occurrence of zero sets is then determined by the interplay between the time derivative, the diffusion term, and the function $f$, as is the case when convection is absent.

Justification of equation (2.4) and estimate (2.5). - It follows from Fubini's theorem that for $u \in H^{1}(\Omega)$ and $\rho_{0}$ such that $B_{\rho_{0}} \subset \Omega$, the function

$$
\rho \longmapsto \int_{\partial B_{\rho}}|\nabla u|^{2}
$$

is defined for almost every $\rho \in\left(0, \rho_{0}\right)$. Since the domain of integration is a cone, we now define the functions $b$ and $E$ in the following way:

$$
b(\rho, t)=\sup _{0 \leq \tau \leq t} \int_{B_{g(\rho, \tau)}} u^{p+1} \text { and } E(\rho, t)=\int_{K_{\rho, t}}|\nabla u|^{2} .
$$

Definition 2.1 guarantees that these expressions are well-defined. It then follows that for almost every $\rho \in\left(0, \rho_{0}\right)$,

$$
\frac{\partial E}{\partial \rho}(\rho, t)=\int_{0}^{t} \int_{\partial B_{g}}|\nabla u|^{2},
$$

and

$$
\int_{0}^{t} \int_{\partial B_{g}} u \mathbf{A}(u, \nabla u) \cdot \nu \leq m_{3}\left(\int_{0}^{t} \int_{\partial B_{g}} u^{2}\right)^{1 / 2}\left(\frac{\partial E}{\partial \rho}\right)^{1 / 2}
$$

We can then combine this with (2.16) to obtain

$$
m_{0} b(\rho, t)+m_{2} E(\rho, t) \leq m_{3}\left(\int_{0}^{t} \int_{\partial B_{g}} u^{2}\right)^{1 / 2}\left(\frac{\partial E}{\partial \rho}\right)^{1 / 2}-\int_{K_{\rho, t}} u f(u, v) .
$$

This inequality is the rigorous counterpart of (2.6).

Handling of the term $\int_{K_{\rho, t}} u f(u, v)$. - Let us now consider the last term in (2.17). Hypothesis (H1) ensures the existence of a function $\psi$. By multiplying the second equation in (1.3) by $\psi(v)$ and integrating we find that

$$
\int_{B_{g(\rho, t)}} \Psi(v(t)) \leq \int_{K_{\rho, t}} \psi(v) f(u, v),
$$




\section{G. Galiano and M. A. Peletier}

for all $0<\rho<\rho_{0}$. Note that since hypothesis (H1) allows the function $\psi(s)$ to assume the value $\infty$ for some values of $s$, the two integrals written above might both be infinite. Now we add this inequality to (2.17) to obtain

$$
\begin{aligned}
& m_{0} b(\rho, t)+\int_{B_{g(\rho, t)}} \Psi(v(t))+m_{2} E(\rho, t) \leq \\
& \leq m_{3}\left(\int_{0}^{t} \int_{\partial B_{g}} u^{2}\right)^{1 / 2}\left(\frac{\partial E}{\partial \rho}\right)^{1 / 2}-\int_{K_{\rho, t}}(u-\psi(v)) f(u, v) .
\end{aligned}
$$

Hypothesis (H1) now ensures that the second term on the left-hand side is non-negative and the last term on the right-hand side is non-positive. This also ensures that both sides of the inequality have finite values. We are left with

$$
m_{0} b(\rho, t)+m_{2} E(\rho, t) \leq m_{3}\left(\int_{K_{\rho, t}} u^{2}\right)^{1 / 2}\left(\frac{\partial E}{\partial \rho}\right)^{1 / 2}
$$

From here onwards the proof is the same as in the formal discussion, with the one exception that Lemma 3.1 should now be applied to a truncated cone instead of a cylinder.

\subsection{Proof of Theorem B}

Throughout the previous section the variable $\rho$ took values in $\left[0, \rho_{0}\right)$. For values outside of this range, i.e., for $\rho \in\left[\rho_{0}, \rho_{1}\right)$, essentially the same arguments hold. The main difference is that when integrating over the cylinder (that is, the passage from (2.2) to (2.3)) the terms at $t=0$ do not necessarily vanish. The equivalent of inequality (2.18) then reads

$$
\begin{aligned}
& m_{0} b(\rho, t)+\int_{B_{\rho}} \Psi(v(t))+m_{2} E(\rho, t) \leq \\
& \quad \leq m_{3}\left(\int_{0}^{t} \int_{B_{\rho}} u^{2}\right)^{1 / 2}\left(\frac{\partial E}{\partial \rho}\right)^{1 / 2}+\int_{B_{\rho}} \Phi\left(u_{0}\right)+\int_{B_{\rho}} \Psi\left(v_{0}\right) .
\end{aligned}
$$

The first term on the right-hand side is handled as before, and the proof of Theorem 1.2 is concluded by the application of Lemma 3.1. 


\subsection{Proof of Theorem C}

The essential difference between Theorem $\mathrm{C}$ and the two other theorems lies in the assumptions on the nonlinearity $f$. We shall therefore only discuss this part of the total argument.

The following lemma combines some technical estimations.

Lemma 2.2. - Let $f$ satisfy (H2) or (H3). Then for all $\varepsilon>0$ and $0<t<T$,

$$
\begin{aligned}
-\int_{0}^{t} \int_{B_{g}} u f(u, v) \leq & -\int_{0}^{t} \int_{B_{g}} u f(u, 0)+ \\
& +\varepsilon^{-1 / \eta} C_{1} t \int_{B_{\rho}} v_{0}^{(\eta+1) / \eta}+\widehat{C}(\varepsilon, t) \int_{0}^{t} \int_{B_{g}} u^{\eta+1}
\end{aligned}
$$

where $\eta=p$ for (H2) and $\eta=\gamma$ for (HS), and

$$
\widehat{C}(\varepsilon, t)=L \varepsilon+\varepsilon^{-1 / \eta} C_{1} t \quad \text { and } \quad C_{1}=C_{1}(\eta, T, L)>0 .
$$

Here $\rho$ takes values in $\left(0, \rho_{0}\right)$ for property FSP and in $\left(0, \rho_{1}\right)$ for property WT.

We first continue the proof of Theorem $\mathrm{C}$ and prove this lemma afterwards.

Let us first tackle the case of property FSP under hypothesis (H2). In that case the integral $\int_{B_{\rho}} v_{0}^{(p+1) / p}$ is equal to zero by assumption. Fix $\eta=p$. Using the non-negativeness of the term $u f(u, 0)$ stated in (H2) we obtain from (2.21)

$$
-\int_{0}^{t} \int_{B_{g}} u f(u, v) \leq \widehat{C} \int_{0}^{t} \int_{B_{g}} u^{p+1} .
$$

Now, combining (2.17) and (2.22) we get

$$
m_{0} b(\rho, t)+m_{2} E(\rho, t) \leq\left(\int_{0}^{t} \int_{B_{g}} u^{2}\right)\left(\frac{\partial E}{\partial \rho}\right)^{1 / 2}+\widehat{C} \int_{0}^{t} \int_{B_{g}} u^{p+1}
$$

By choosing $\varepsilon$ and $t^{*}$ small enough,

$$
\widehat{C}(\epsilon, t) t \leq \frac{1}{2} m_{0} \quad \text { for all } t \in\left[0, t^{*}\right] .
$$


Then (2.23) becomes

$$
\frac{1}{2} m_{0} b(\rho, t)+m_{2} E(\rho, t) \leq\left(\int_{0}^{t} \int_{\partial B_{g}} u^{2}\right)\left(\frac{\partial E}{\partial \rho}\right)^{1 / 2},
$$

and we conclude by a combination of Theorem 3.2 and Lemma 3.1, in the same way as in the proof of Theorem A.

For property WT we consider cylinders $B_{\rho} \times(0, t)$ where $\rho$ now takes values in $\left(0, \rho_{1}\right)$, which introduces two extra terms in (2.24):

$$
\begin{aligned}
\frac{1}{2} m_{0} b(\rho, t)+m_{2} E(\rho, t) \leq & \left(\int_{0}^{t} \int_{\partial B_{\rho}} u^{2}\right)^{1 / 2}\left(\frac{\partial E}{\partial \rho}\right)^{1 / 2}+ \\
& +m_{1} \int_{B_{\rho}} u_{0}^{p+1}+\varepsilon^{-1 / \eta} e^{C_{1} T} L t \int_{B_{\rho_{0}}} v_{0}^{(\eta+1) / \eta}
\end{aligned}
$$

The result follows in the same way as in the proof of Theorem B.

When we trade hypothesis (H2) for hypothesis (H3) we introduce a new energy,

$$
c(\rho, t) \stackrel{\text { def }}{=} \frac{1}{1+\gamma} \int_{K_{\rho, t}} u^{\gamma+1} .
$$

Using (H3) and (2.21), inequality (2.17) becomes

$$
\begin{aligned}
& m_{0} b(\rho, t)+m_{2} E(\rho, t)+\left(k_{2}-\widehat{C}(\varepsilon, t)\right) c(\rho, t) \leq \\
& \quad \leq m_{3}\left(\int_{0}^{t} \int_{\partial B_{g}} u^{2}\right)^{1 / 2}\left(\frac{\partial E}{\partial \rho}\right)^{1 / 2}+\varepsilon^{-1 / \gamma} e^{C_{1} T} L t \int_{B_{\rho}} v_{0}^{(\gamma+1) / \gamma} .
\end{aligned}
$$

For property FSP the last term disappears, and we choose $\varepsilon$ and $t^{*}$ such that $\widehat{C}(\varepsilon, t) \leq k_{2} / 2$ for all $0 \leq t \leq t^{*}$. Then, applying Theorem 3.2 with parameter $\gamma$ instead of $p$, we obtain

$$
\left(\int_{0}^{t} \int_{\partial B_{g}} u^{2}\right)^{1 / 2}\left(\frac{\partial E}{\partial \rho}\right)^{1 / 2} \leq \varepsilon(E+c)+\frac{C}{\varepsilon}\left(t^{(1-\theta) / 2}\right)^{1 /(1-\kappa)}\left(\frac{\partial E}{\partial \rho}\right)^{1 / \beta}
$$

where $\kappa, \theta$ and $\beta$ all have the same values as in (2.7), (2.8) and (2.10) with $p$ replaced by $\gamma$, and

$$
K(T)=\max \left(1, T^{\theta / 2}\right) \max \left(1, c\left(T, \rho_{1}\right)^{\theta(1-\gamma) / 2(\gamma+1)}\right) .
$$


Notably, $\beta$ has the value

$$
\beta=\frac{3 \gamma+1+N(1-\gamma)}{2 \gamma+2+N(1-\gamma)}
$$

and therefore $\beta<1$ if and only if $\gamma<1$. Property WT is handled analogously.

We conclude with the proof of Lemma 2.2. Multiply the second equation in (1.3) by $v^{q-1}$, where $q>1$ will be fixed later. Integrating over $B_{g(\rho, t)}$ and using formula (2.15) we obtain

$$
\frac{1}{q} \frac{\mathrm{d}}{\mathrm{d} t} \int_{B_{g}(\rho, t)} v^{q} \leq \int_{B_{g}(\rho, t)} v^{q-1} f(u, v) .
$$

Now, if we write

$$
f(u, v)=f(u, 0)+(f(u, v)-f(u, 0))
$$

and use the assumption of Lipschitz continuity of $f$ in $v$ together with either (H2) or (H3) - depending on which one is valid - we find

$$
\begin{aligned}
\int_{B_{g}(\rho, t)} v^{q-1} f(u, v) & \leq \int_{B_{g}(\rho, t)} v^{q-1} f(u, 0)+L \int_{B_{g}(\rho, t)} v^{q} \\
& \leq C \int_{B_{g}(\rho, t)} v^{q-1} u^{\eta}+L \int_{B_{g}(\rho, t)} v^{q}
\end{aligned}
$$

and with Young's inequality with exponent $q$ we obtain

$$
\int_{B_{g}(\rho, t)} v^{q-1} f(u, v) \leq\left(L+C \frac{q-1}{q}\right) \int_{B_{g}(\rho, t)} v^{q}+\frac{C}{q} \int_{B_{g}(\rho, t)} u^{\eta q},
$$

where:

- for (H2) we set $\eta=p$ and $C=k_{1}$, and

- for (H3) we set $\eta=\gamma$ and $C=k_{3}$.

By Gronwall's Lemma it follows that (setting $C^{\prime}=(q-1) C$ )

$$
\int_{B_{g(\rho, t)}} v^{q}(t) \leq e^{C^{\prime} t} \int_{B_{\rho_{0}}} v_{0}^{q}+C \int_{0}^{t} e^{C^{\prime}(t-\tau)} \int_{B_{g(\rho, \tau)}} u^{\eta q}(\tau) \mathrm{d} x \mathrm{~d} \tau .
$$




\section{G. Galiano and M. A. Peletier}

Using this estimate on $v$, we estimate the integral of $u f(u, v)$ which appears in (2.17). Again using the decomposition $f(u, v)=f(u, 0)+$ $(f(u, v)-f(u, 0))$, the Lipschitz continuity, and Young's inequality, we obtain for any $\epsilon>0$,

$$
\begin{aligned}
& -\int_{0}^{t} \int_{B_{g}} u f(u, v) \leq \\
& \leq-\int_{0}^{t} \int_{B_{g}} u f(u, 0)+L \int_{0}^{t} \int_{B_{g}} u v \\
& \leq-\int_{0}^{t} \int_{B_{g}} u f(u, 0)+\varepsilon L \int_{0}^{t} \int_{B_{g}} u^{\eta+1}+\varepsilon^{-1 / \eta} L \int_{0}^{t} \int_{B_{g}} v^{(\eta+1) / \eta},
\end{aligned}
$$

where we are writing again $g$ for $g(\rho, \tau)$. Now if we use (2.27) with $q=(\eta+1) / \eta$ we obtain

$$
\begin{aligned}
-\int_{0}^{t} \int_{B_{g}} u f(u, v) \leq & -\int_{0}^{t} \int_{B_{g}} u f(u, 0)+\varepsilon L \int_{0}^{t} \int_{B_{g}} u^{\eta+1}+ \\
& +\varepsilon^{-1 / \eta} e^{C^{\prime} T} L t \int_{B_{\rho_{0}}} v_{0}^{q}+\varepsilon^{-1 / \eta} e^{C^{\prime} T} L C t \int_{0}^{t} \int_{B_{g}} u^{\eta+1}
\end{aligned}
$$

and by rearranging the different terms,

$$
\begin{aligned}
-\int_{0}^{t} \int_{B_{g}} u f(u, v) \leq & -\int_{0}^{t} \int_{B_{g}} u f(u, 0)+\varepsilon^{-1 / \eta} e^{C^{\prime} T} L t \int_{B_{\rho_{0}}} v_{0}^{q}+ \\
& +L\left(\varepsilon+\varepsilon^{-1 / \eta} C t e^{C^{\prime} T}\right) \int_{0}^{t} \int_{B_{g}} u^{\eta+1}
\end{aligned}
$$

This proves the lemma.

\section{Appendices}

\subsection{Physical background}

One of the major hypotheses that we use in this paper (hypothesis (H1)) has a mathematically unusual form. When interpreted in physical terms, however, this hypothesis is very natural and even arises directly in the model derivation. We therefore feel that the reader may benefit from a brief 
discussion of the physical background of (1.1). Since our interest lies in the structure, not the details, of the derivation of the model, we shall simply explain the underlying ideas without aspiring to physical completeness.

We are interested in the transport of a hydrophobic chemical through groundwater flow. This chemical substance can be found in either of two states: dissolved in the water and adsorbed on the surface of the soil particles. Let $C$ and $S$ denote the concentration of the chemical in dissolved and adsorbed form, respectively. It is common in the hydrological literature to assume incompressibility,

$$
\operatorname{div} \mathbf{w}=0
$$

and conservation of mass,

$$
(C+S)_{t}+\operatorname{div}(C \mathbf{w}-D \nabla C)=0
$$

where $\mathbf{w}$ is a given discharge field and $D$ is a combined dispersion/diffusion tensor. In (3.1) the assumption is implicitly present that the chemical substance only moves in dissolved state.

The behaviour of the concentrations $C$ and $S$ is mainly determined by the choice of the interaction between them. A typical assumption is

$$
S_{t}=k f(C, S),
$$

where $f$ is called the rate function and $k>0$ is a rate parameter. It is also common to distinguish between different types of adsorption sites on the surface of the soil particles. This leads to a formulation of the form

$$
\left\{\begin{array}{l}
S=\sum S_{i} \\
S_{i t}=k_{i} f_{i}\left(C, S_{i}\right) .
\end{array}\right.
$$

In experimental practice it is not easy to measure the rate function $f$. However, a very natural quantity to measure is the set of pairs $(C, S)$ such that $f(C, S)=0$. If this set can be represented as the graph of a function $\varphi$, i.e.,

$$
f(C, S)=0 \Longleftrightarrow S=\varphi(C),
$$

then $\varphi$ is called an isotherm. The curve $S=\varphi(C)$ divides the $S, C$-plane into two parts; a common physical observation is that

$$
\begin{array}{ll}
f(C, S)<0 & \text { when } S>\varphi(C) \\
f(C, S)>0 & \text { when } S<\varphi(C) .
\end{array}
$$




\section{G. Galiano and M. A. Peletier}

In view of (3.2) this property can be interpreted as stating that the chemical always seeks an equilibrium distribution. In some sense it therefore is a stability property.

By setting $u=C$ and $v=S$, equations (3.1) and (3.2) form a system of the same type as (1.1). In this case the function $\phi$ can be taken to be the identity. In the sequel we shall also be interested in cases in which $\phi$ has an unbounded derivative in the origin, and we shall therefore briefly show how such a situation follows from similar assumptions.

Consider a model that allows for two types of adsorption sites: sites of type 1 are considered to react on the same time scale as the advection dynamics, while sites of type 2 are supposed to react much more quickly. We can therefore reasonably assume that for sites of type 2 the equilibrium condition $S_{2}=\varphi_{2}(C)$ is satisfied at all time, which allows us to substitute this expression into (3.1):

$$
\left(C+\varphi_{2}(C)+S_{1}\right)_{t}+\operatorname{div}(C \mathbf{w}-D \nabla C)=0 .
$$

For $S_{1}$ we retain the original equation

$$
S_{1 t}=f_{1}\left(C, S_{1}\right)
$$

By setting $u=C$ and $v=S_{1}$ we now obtain a nonlinear function $\phi$ that depending on the isotherm $\varphi_{2}$ - may or may not degenerate at $C=0$.

We now return to hypothesis (H1). Expressed in terms of $C$ and $S_{1}$, this hypothesis states that:

$$
\left\{\begin{array}{l}
\text { there exists a function } \psi \text { such that } \\
\quad\left(C-\psi\left(S_{1}\right)\right) f_{1}\left(C, S_{1}\right) \geq 0 \\
\text { for all } C \text { and } S_{1}
\end{array}\right.
$$

It follows from property (3.3) that if the isotherm $\varphi_{1}$ is invertible, we can choose its inverse $\varphi_{1}^{-1}$ as the function $\psi$ in (3.4). Generally, experimentally determined isotherms are monotone and therefore invertible, although other cases have been observed. 


\subsection{A nonlinear ordinary differential inequality}

Lemma 3.1. - Let $\gamma \geq 0$ and $0<\beta<1$.

1) Let $\delta \geq 0$ and let $\varphi \in C\left(\left[0, \rho_{0}+\delta\right] \times[0, T]\right)$ be a nonnegative function such that

$$
\varphi^{\beta}(\rho, t) \leq K t^{\gamma} \frac{\partial \varphi}{\partial \rho}(\rho, t)+\varepsilon\left(\rho-\rho_{0}\right)_{+}^{\beta /(1-\beta)}
$$

for almost every $\rho \in\left[0, \rho_{0}+\delta\right]$ and for all $t \in[0, T]$. Here $\varepsilon$ is a nonnegative number. If $\varphi$ is non-decreasing in both arguments, then there exists a time $0<t^{*} \leq T$ such that $\varphi=0$ on $\left[0, \rho_{0}\right] \times\left[0, t^{*}\right]$.

2) Let $K_{\rho_{0}, T}$ be the cone defined in (2.14) with $\rho=\rho_{0}$ and $t=T$, and let $\varphi \in C\left(\overline{K_{\rho_{0}, T}}\right)$ be a nonnegative function such that

$$
\varphi^{\beta}(\rho, t) \leq K t^{\gamma} \frac{\partial \varphi}{\partial \rho}(\rho, t)
$$

for all $t \in[0, T]$ and for almost all $\rho \in\left[0, g\left(\rho_{0}, t\right)\right]$. Then there exists a continuous function $r:[0, T] \rightarrow \mathbb{R}$ with $r(0)=\rho_{0}$ such that

$$
\varphi(\rho, t)=0 \text { for all } \rho \text { and } t \text { such that } \rho \leq r(t) .
$$

Proof. - For part 1), we consider an auxiliary function $z=z(\rho)$ that satisfies

$$
z^{\beta}(\rho)=K\left(t^{*}\right)^{\eta} \frac{\mathrm{d} z}{\mathrm{~d} \rho}(\rho)+\varepsilon\left(\rho-\rho_{0}\right)_{+}^{\beta /(1-\beta)}
$$

and

$$
z\left(\rho_{0}+\delta\right) \geq \varphi\left(\rho_{0}+\delta, t^{*}\right) .
$$

Here $t^{*}>0$ is still to be chosen. It is easily shown that the function

$$
z(\rho)=A\left(\rho-\rho_{0}\right)_{+}^{1 /(1-\beta)}
$$

satisfies these two conditions if

$$
A>\max \left\{\varepsilon^{1 / \beta}, \varphi\left(\rho_{0}+\delta, t^{*}\right) \delta^{-1 /(1-\beta)}\right\} .
$$

In that case $t^{*}$ is deduced from (3.6):

$$
A^{\beta}=K\left(t^{*}\right)^{\eta} \frac{A}{1-\beta}+\varepsilon .
$$

The statement of the lemma then follows from the monotonicity of $\varphi$ in $t$ and $\rho$. 


\section{G. Galiano and M. A. Peletier}

Part 2) is proved in the following way. Fix $0<t \leq T$ and suppose that $\varphi$ is strictly positive on $\left(\underline{\rho}, g\left(\rho_{0}, t\right)\right]$. Remark that by $(3.5)$ the function $\varphi$ is non-decreasing in $\rho$, and therefore $\varphi(\rho, t)>0$ implies $\varphi(\cdot, t)>0$ on $\left(\rho, g\left(\rho_{0}, t\right)\right]$. Then for all $\underline{\rho}<\rho \leq g\left(\rho_{0}, t\right)$,

$$
(1-\beta) K^{-1} t^{-\gamma} \leq \frac{\partial\left(\varphi^{1-\beta}\right)}{\partial \rho} .
$$

Now integrate over $\left(\underline{\rho}, g\left(\rho_{0}, t\right)\right]$ :

$$
(1-\beta) K^{-1} t^{-\gamma}\left(g\left(\rho_{0}, t\right)-\underline{\rho}\right) \leq \varphi^{1-\beta}\left(g\left(\rho_{0}, t\right), t\right)-\varphi^{1-\beta}(\underline{\rho}, t),
$$

or equivalently,

$$
\varphi^{1-\beta}(\underline{\rho}, t) \leq \varphi^{1-\beta}\left(g\left(\rho_{0}, t\right), t\right)-(1-\beta) K^{-1} t^{-\gamma}\left(g\left(\rho_{0}, t\right)-\underline{\rho}\right) .
$$

Clearly this implies a contradiction if

$$
\underline{\rho}<g\left(\rho_{0}, t\right)-\frac{K}{(1-\beta)} t^{\gamma} \varphi^{1-\beta}\left(g\left(\rho_{0}, t\right), t\right) .
$$

Since $\varphi$ is continuous on the closed set $\bar{K}_{\rho_{0}, T}, \varphi^{1-\beta}$ is bounded by a constant $M>0$ and as a consequence

$$
\varphi(\rho, t)=0 \quad \text { if } \rho \leq g\left(\rho_{0}, t\right)-\frac{K M}{(1-\beta)} t^{\gamma} .
$$

\subsection{An interpolation-trace inequality}

As there exist some slighty different versions of interpolation-trace inequalities, we present here that used in the present work. For further reference (see [1], [9]). We denote the norm of the space of Lebesgue integrable functions $L^{p}(X)$ on a measure space $X$ by $\|\cdot\|_{p, X}$.

THEOREM 3.2. - Let $\Omega$ be a bounded domain in $\mathbb{R}^{N}$ with piecewise smooth boundary $\Gamma$ and let $u \in W^{1, p}(\Omega), 1<p<\infty$. The following inequality holds:

$$
\begin{gathered}
\|u\|_{q, \Gamma} \leq C\left(\|\nabla u\|_{p, \Omega}+\|u\|_{\gamma, \Omega}\right)^{\theta}\|u\|_{r, \Omega}^{1-\theta} \\
-440-
\end{gathered}
$$


where

$$
\begin{gathered}
\theta=\frac{p}{q} \frac{q N-r(N-1)}{p(N+r)-N r} \in(0,1), \quad 1 \leq \gamma<\infty \\
1 \leq r<\frac{N p}{N-p} \text { and } 1 \leq q<\frac{p(N-1)}{N-p} \text { if } N>p \\
1 \leq r, q<\infty \text { if } p=N, \\
1 \leq r, q \leq \infty \text { if } p>N
\end{gathered}
$$

and the constant $C$ depends on $\Omega$ and the exponents.

\section{References}

[1] Antontsev (S.) and Díaz (J. I.) .- Energy Methods for Free Boundary Problems in Continuum Mechanics, To appear.

[2] Antontsev (S.), Díaz (J. I.) and Shmarev (S. I.) .- The support shrinking properties for local solutions of quasilinear parabolic equations with strong absorption terms, Ann. Fac. Sciences de Toulouse 4, $n^{\circ} 1$ (1995), pp. 5-30.

[3] Antontsev (S. N.) .- On the localization of solutions of nonlinear degenerate elliptic and parabolic equations, Dokl. Akad. Nauk. SSSR, 260, $\mathrm{n}^{\circ} 6$ (1981), pp. 1289-1293 (in Russian; English translation in Soviet Math. Dokl. $24 \mathrm{n}^{\circ} 2$ (1981), pp. 420-424).

[4] BERNIS (F.) .- Finite speed of propagation and continuity of the interface for thin viscous films, Adv. Diff. Eqns 1, ${ }^{\circ} 3$ (1996), pp. 337-368.

[5] DíAz (J. I.) and STAKGOLD (I.) .- Mathematical aspects of the combustion of a solid by a distributed isothermal gas reaction, S.I.A.M. J. Math. Anal. 26, $\mathrm{n}^{\circ} 2$ (1995), pp. 305-328.

[6] Díaz (J. I.) and Veron (L.) .- Local vanishing properties of solutions of elliptic and parabolic quasilinear equations, Trans. A.M.S. 290, $\mathbf{n}^{\circ} 2$ (1985), pp. 787-814.

[7] DuiJn (C. J. VAN) and KNABNER (P.) . - Solute transport in porous media with equilibrium and non-equilibrium multiple-site adsorption: Travelling waves, J. reine angew. Math. 415 (1991), pp. 1-49.

[8] Hilhorst (D.), van Der Hout (R.) and Peletier (L. A.) .- Diffusion in the presence of fast reaction: The case of a general monotone reaction term, J. Math. Sci. Tokyo 4, $\mathbf{n}^{\circ} 3$ (1997), pp. 469-517.

[9] Ladyženskaya (O. A.), Solonnikov (V. A.) and Ural'Ceva (N. N.) .Linear and Quasi-linear Equations of Parabolic Type, Translations of Mathematical Monographs, A.M.S. 23 (1968).

[10] SHMAREV (S. I.) .- Local properties of solutions of nonlinear equation of heat conduction with absorption, Dinamika Sploshnoi Sredy, Novosibirsk 95 (1990), pp. 28-42. 